\title{
A Limping Child: On A First Look It Is Hip Or Knee In Origin! No! It's A Talus Osteomyelitis
}

\author{
P Ganaisan, A Singh, C Kumar, R Manikam
}

\begin{abstract}
Citation
P Ganaisan, A Singh, C Kumar, R Manikam. A Limping Child: On A First Look It Is Hip Or Knee In Origin!' No! It's A Talus Osteomyelitis. The Internet Journal of Orthopedic Surgery. 2013 Volume 20 Number 1.
\end{abstract}

DOI: $\underline{10.5580 / 2 \mathrm{cde}}$

\begin{abstract}
Isolated osteomyelitis of the talus in a child is very rare incidence [1,2]. Children presenting with pain and limp to emergency department are predominantly investigated for pathologies of hip or knee, hence a condition originating from talus is frequently missed. A delay in its diagnosis are precursors to gait and bony deformity in future where the cost of disability is a huge burden to the patient. We present a case of osteomyelitis of talus where initially it was a missed diagnosis but due to awareness and alertness of the treating physician it was identified and treated early.
\end{abstract}

\section{CASE REPORT}

A 2 year old child presented to Emergency Department with pain and swelling of her left foot and ankle for 1 month. Her parents claimed the child had sustained what was initially thought as a superficial injury to the foot about 6 weeks earlier- hit by a stone while playing. Seen and treated by a general practitioner - no radiographs were done then. Over the subsequent month she experienced increasing swelling and pain- affecting mobility, with an occasional fever. During the initial admission, the foot was warm, tender and swollen till the ankle. There was no neurovascular deficit, open wounds or discharging sinus. Based on radiographic and clinical findings, a diagnosis of crack fracture of the left talus dome with overlying cellulites was made - secondary to the initial trauma . A broad spectrum antibiotic was started and the limb immobilized in a below knee backslab cast. She was discharged home after 2 days as the constituitional symptoms had resolved and cellulitis appeared to respond to the treatment- a review planned a week later. By the next follow-up the child could weight bear, the pain and erythema had subsided but swelling persisted. The antibiotics were continued for another week, and a 3 weekly review planned. At 9 weeks, in view of the persistent swelling and lytic changes obvious on radiographs a MRI was planned. Differentials were AVN and Osteomyelitis. The MRI, done 12 weeks from date of admission ( 18 weeks post trauma) was reported as Chronic Osteomyelitis with underlying pathological fractures.

\section{Figure 1}

Figure1. Radiographic changes at 9wks

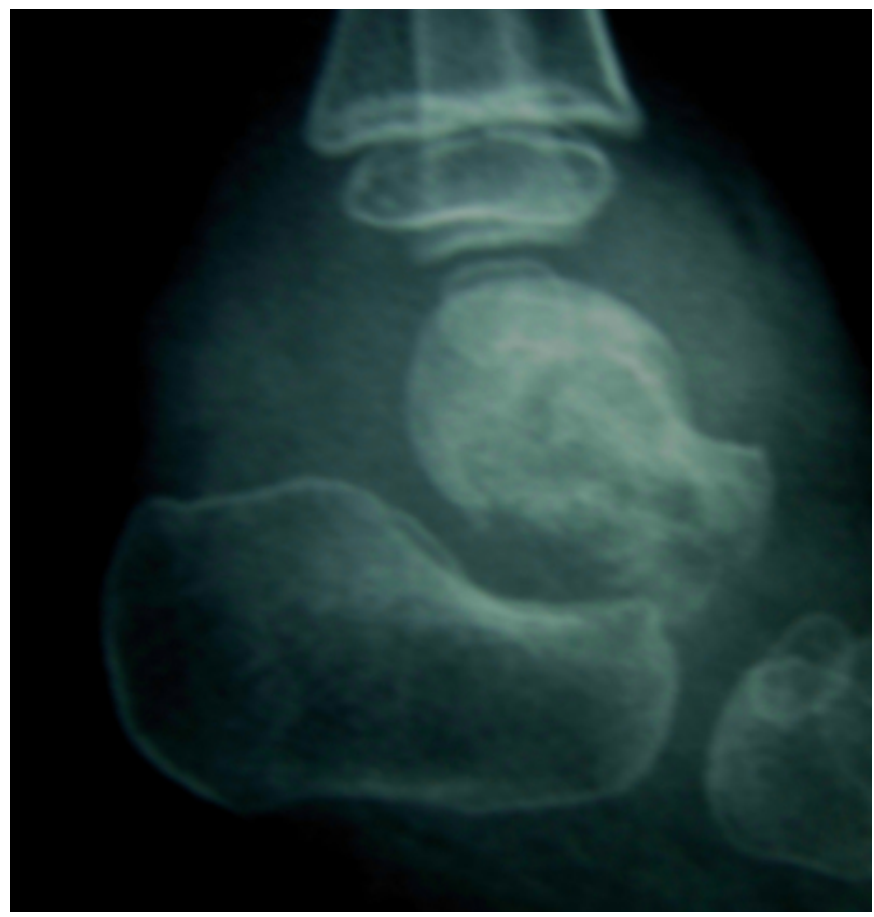




\section{Figure 2}

Figure 2. MRI changes at $12 \mathrm{wks}$

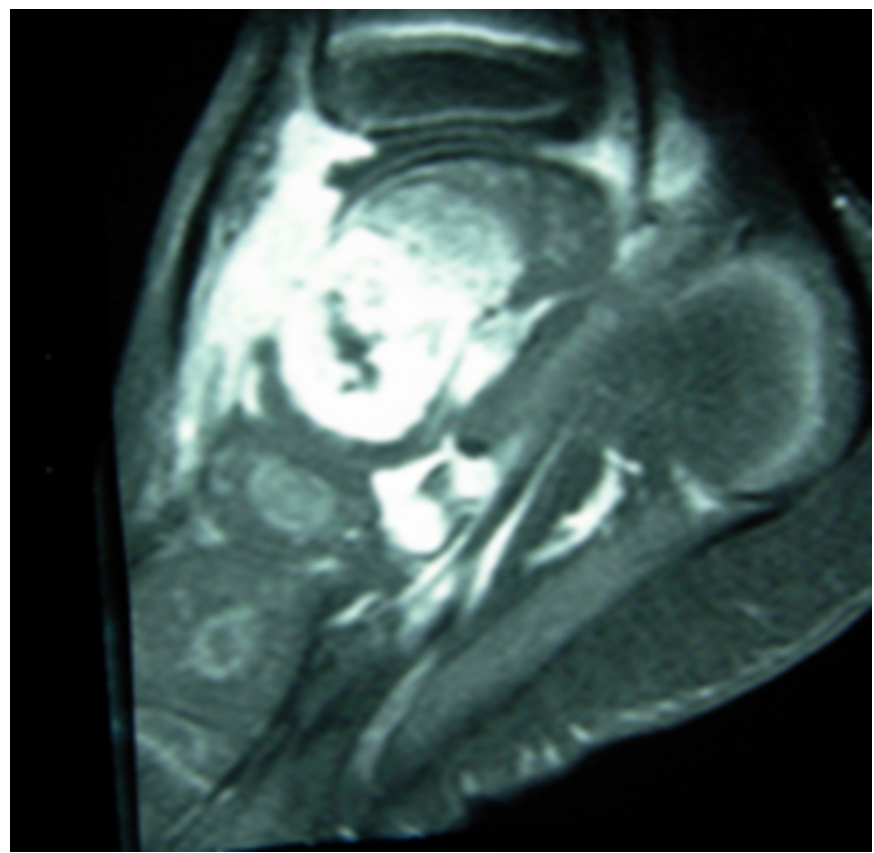

Sequestrectomy preformed 3 weeks later produced necrotic bone and a serosanginous fluid. Culture and sensitivity of fluid and bone sample were negative for any organism. HPE confirmed osteomyelitis. Post operative reviews at 1 week and 3 weeks showed a resolving swelling and radiographic evidence of healthy callus. She was discharged well at 6 weeks ( 28 weeks post trauma) without a bony defect or gait deformity. A yearly review planned.

\section{Figure 3}

Figure. 3 Healing callus seen 6 weeks post-operatively

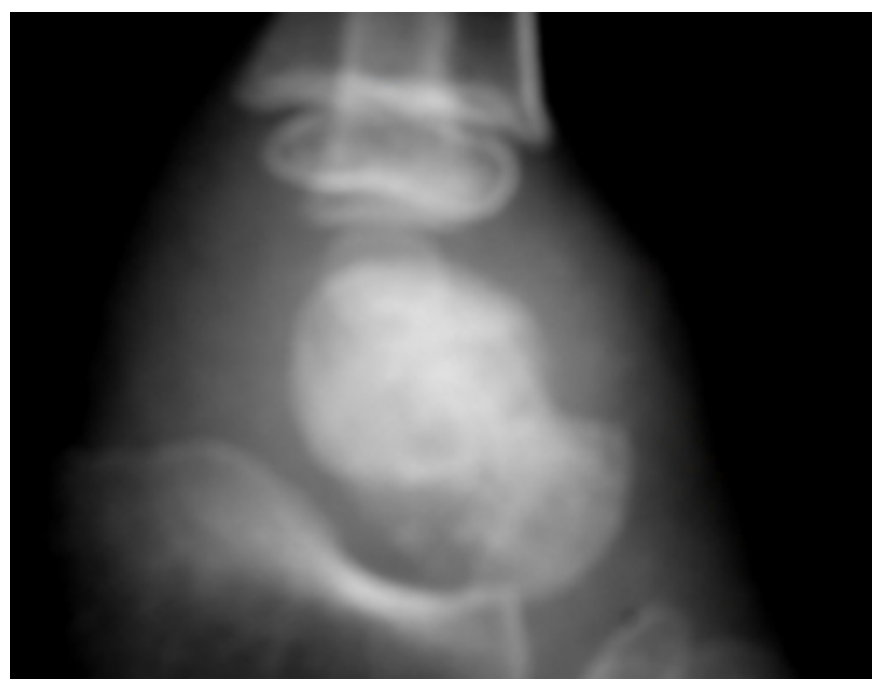

\section{Figure 4}

Table I. Laboratory findings

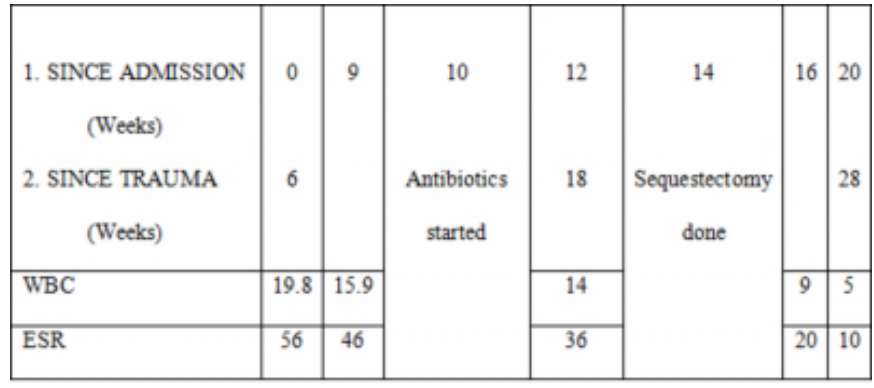

ESR- erythrocyte sedimentation rate ( $\mathrm{mm} / \mathrm{hr})$; normal (0-10)

WBC- white blood cells ( cells mm3 ) ; normal (4-11 x 10)

\section{DISCUSSION}

Primarily the hip or the knee joint comes as the main anatomic locations of an limping child in the mind of a treating medical doctor. The above case reiterates the fact that osteomyelitis of the talus is a rarely thought of diagnosis in limping child. Nixon [4], attributed similarity of osteomyelitic spread in the talus with long metaphyseal bones to its anatomic subdivisions .These anatomic subdivisions with their individual vascular supply form metaphyseal type locations in the talus, thus increasing chances of infection. Documented cases have been either acute or subacute, with chronicity mainly due to delays in diagnosis. Antoniou and Connor (1974) used the criteria of duration of symptoms with definitive radiographic and clinical findings as a guideline - acute if the signs and symptoms appeared within two weeks and subacute if case history longer than two weeks. Frequent differentials include ligament injuries or fractures. Common causes for the failure in its early diagnosis include the radiographic changes begin around 10-12 weeks [5], hence immediate films may be insignificant; also the soft tissue changes mimic any ligament or superficial infection $[1,2,3]$. Furthermore, primary subacute osteomyelitis may present the above features minus the fever; hence diagnosis would be delayed till after bony destruction has occurred [3]. We advocate a high index of suspicion by the treating primary doctor .A more organised regular follow-up at an orthopaedic clinic is arranged .

\section{References}

1. Grattan-smith,JD, Wagner, ML .Osteomyelitis of the talus-unusual case of the limping child. AJR 1991:785-789 2. Antionou, D, Conner, AN. Osteomyelitis of the calcaneus and talus. JBJS (Am) 1974; 56-A: 338-345

3. Skevis, XA. Primary subacute osteomyelitis of the talus. JBJS (Br) 1984; 66-B:101-103

4. Nixon, GW. Haematogenous osteomyelitis of 
metaphyseal-equivalent locations. AJR 1978; 130:123-129

observations in osteomyelitis. AJR 1978; 108:488-496

5. Capitanio, MA, Kirkpatrick, JA. Early roentgen 


\section{Author Information}

Premganesh Ganaisan, MBBS

Department Of Orthopaedics, University Malaya

\section{Avthar Singh, MBBS, MS Ortho}

Department Of Orthopaedics, University Malaya

\section{CS Kumar, MBBS , MS Ortho}

Department Of Orthopaedics, University Malaya

\section{Rishya Manikam, MBBS , M.EMMED}

Head, Department Of Emergency Medicine, University Malaya 